\title{
A simple and inexpensive startle transducer with high output
}

\author{
DONALD S. LEITNER \\ Columbia University, New York, New York \\ and \\ MATTHEW C. ROSENBERGER \\ New York State Psychiatric Institute, New York, New York
}

\begin{abstract}
A piezoelectric film material laminated to a sheet of flexible Plexiglas shows promise as a simple, high-performance startle trangducer. It is an improvement over many other startle transducers because of its high output, insensitivity to vibrational and electrical artifacts, simplicity of instrumentation, and modest cost. Although discussed in terms of its application to measurement of the startle response, the material has potential for use in many situations that require a movement transducer.
\end{abstract}

The startle response in mammals is elicited by intense sensory stimulation with an abrupt onset (Fleshler, 1965). This response has been used as a reliable, easily elicited measure in a large number of diverse experimental applications, ranging from electrophysiology to learning and sensory evaluation. Most devices used to quantify the startle response in rats utilize a small cage that is mounted to a rigid superstructure by a damped mounting system. The abrupt movements that characterize the startle response cause a displacement of the cage relative to the superstructure, whereas less abrupt movements, such as those involved in locomotion or grooming, are not readily transmitted.

Several different transducers have been developed to measure the cage's displacement. The simplest of these is a magnet that is mounted to the cage adjacent to a coil of wire that is affixed to the superstructure (e.g., Hoffman \& Fleshler, 1964). The cage's displacement moves the magnet relative to the coil of wire, generating an electrical current that is proportional to the rate of movement. The output from the coil of wire can be measured on an oscilloscope, strip-chart recorder, etc., but usually requires amplification and filtering. A similar, more sophisticated startle transducer replaces the wire

This research was supported by National Institutes of Health Grants MH-15174 and R01-NS18822 and by Biomedical Research Support Grant 903-E579P. The authors would like to thank J. V. Chatigny, sales manager for the Piezo Film Department of the Pennwalt Corporation, for his helpful suggestions. Reprint requests should be addressed to: Donald S. Leitner, Department of Behavioral Physiology, Columbia University, 722 West 168th Street, Box 115, New York City, New York 10032 . coil with a solid-state, Hall-effect proximity sensor (Foss \& Ison, Note 1). ${ }^{1}$ Movement of the magnet near the sensor (Microswitch 634SS2) produces a voltage change that is proportional to the change in Gauss and that can be filtered, amplified, and measured as above.

Both of these transducers are quite sensitive and reliable. Because of their low output, however, amplification of the signal is necessary, increasing the amount of instrumentation needed to obtain data. The low output also makes the signal susceptible to electrical noise and interference, which requires filtering of the signal, a further complication. Also, these devices tend to be susceptible to building vibration, stimulus artifacts, and other extraneous artifacts. Finally, the Hall-effect sensor is a powered device, requiring a regulated power supply to operate.

Another device is an accelerometer (e.g., White \& Horlington, 1969). Attached to the cage itself, it has an output voltage proportional to the velocity of the cage's displacement. Although sensitive, reliable, and not dependent on a relative displacement, the transducer and its associated electronics are expensive.

A piezoelectric film material shows promise as an inexpensive, reliable, high-output transducer for the measurement of startle. This material, when stretched, produces a voltage proportional to the magnitude of the stretch. When mounted as described in this paper, it has a high output voltage, requires no filtering or amplification, is insensitive to vibrational and electrical artifacts, requires no power supply, is self-contained, and is inexpensive. Although the focus of the present article is on this material's use as a startle transducer, it potentially could be adapted to measure other types of small-animal movement. 


\section{PIEZOELECTRIC FILM STARTLE TRANSDUCER}

A $10 \times 20 \mathrm{~cm}$ piece of a piezoelectric film material (Kynar, Pennwalt) was obtained from the Piezo Film Department of the Pennwalt Corporation, 900 First Avenue, P.O. Box C, King of Prussia, Pennsylvania 19406. The sample had a nominal thickness of 28 microns and cost $\$ 35$. Thicker pieces have a higher output, but cost more. For a given thickness, smaller pieces are less expensive, but also provide less output. Amplification may be required if small pieces are used.

The material resembles a light-gauge, flexible sheet of aluminum foil. It must be handled carefully, for it is rather easily damaged. The two faces of the material are its poles, and anything that puts the two poles in direct contact with each other essentially produces a short circuit. Anything that punctures the material can produce such a short circuit. Because of this, care must be exercised in working with the material to keep metal filings, plastic chips, etc., away from the material.

The material cannot be soldered. Electrical contacts are made by first soldering short lengths of 24-ga wire to copper foil tabs that have conductive adhesive backing, which are supplied with the sample. Then, one tab is affixed to each face of the material and is held in place by the adhesive backing. Other methods of making electrical contact are discussed in the applications note supplied with the material.

In our preparation, after the electrical contacts had been made the material, with its two leads attached, was then laminated to a piece of clear Plexiglas (32.5 $\times 19.5$ $x 0.5 \mathrm{~cm}$ ) with Devcon 5 Minute epoxy. This was done by applying a thin layer of epoxy to the material and a thin layer of hardener to the Plexiglas. The material was then centered and firmly pressed onto the Plexiglas sheet, and the epoxy was allowed to set. When the epoxy had fully cured, two binding posts were mounted on the Plexiglas, away from the piezo film. The ends of the leads from the film were soldered to the binding posts.

The Plexiglas was then mounted to a rigid superstructure that suspended it $20 \mathrm{~cm}$ above the floor of an experimental chamber, and the top of a small cage was mounted to the underside of the Plexiglas so that it, too, was suspended with the Plexiglas supporting it. The Plexiglas was oriented so that the piezo film lay between the top of the cage and the Plexiglas, to protect the transducer material. The cage was centered on the Plexiglas sheet so that, if it moved, it maximized the flexion of the Plexiglas. As the Plexiglas flexed, it stretched the attached piezo film material and produced a voltage measurable across the binding posts.

\section{PERFORMANCE}

Piezoelectric devices are charge generators, and as such their output voltage is a function of the capacitance of the system in which they are used. Different types and lengths of cable used to connect the transducer to a recording device will therefore affect the output voltage. In the present case, $30 \mathrm{~m}$ of 24 -ga two-conductor shielded microphone cable were used to connect the transducer via its binding posts to various measurement devices. The shield is connected to ground.

The output from the transducer was monitored on an oscilloscope with storage capability (Tektronix Model 564B, with a Type 3A74 plug-in amplifier and a Type 2B67 plug-in time base). When tapped with a finger, the transducer produced a sinusoidal waveform 40 to $80 \mathrm{~Hz}$ in frequency, with a magnitude that varied with the force of the tap. The peak voltage ranged from approximately 0.5 to approximately $3 \mathrm{~V}$.

The transducer was then tested to ascertain the linearity of its output with respect to the amount of force applied to it. The stability of the transducer's output over time was also examined. The cage was loaded with a $350 \mathrm{~g}$ weight to simulate the presence of a rat, and a 1.2-g sphere fashioned from orthodontic acrylic was dropped upon the top of the cage from varying heights. The output from the transducer was fed directly into a Coldspring Instrument Corporation $A / D$ voltage converter (Model 17D). This device samples an incoming waveform for an externally controllable period of time and converts the highest deflection above zero into a stream of pulses measurable on an electromechanical counter. The number of pulses is proportional to the peak input voltage. The sampling window of the device was controlled by a Grass stimulator (Model S88) operated manually. The sample window was opened for $5 \mathrm{sec}$, beginning at the time that the sphere was dropped.

The acrylic sphere was dropped 10 times from each of 10 heights, ranging from 10 to $100 \mathrm{~cm}$, in $10-\mathrm{cm}$ increments. The mean output from the transducer was computed across the 10 trials at each drop height, and each mean was converted back into volts.

This procedure was performed three times over 5 weeks, allowing 2 weeks to elapse between replications. To examine the linearity of the output, means were computed across the three sessions for each drop height. These data are plotted in Figure 1. It is apparent that the output voltage of the transducer was linear across the range tested. This was confirmed by a Pearson product-moment correlation coefficient computed on these data, which demonstrated that a strong linear relationship existed between drop height and transducer output $[\mathrm{r}(8)=.988, \mathrm{p}<.001]$.

To test the stability of the transducer over time, the data for each of the three calibration sessions were collapsed across drop heights, and a one-way analysis of variance for repeated measures was performed across test days. This analysis revealed no reliable change in output voltage over time $[F(2,18)=2.13$, n.s. $]$. These data indicate that the transducer's output was stable across the three sessions.

The performance of the transducer was also tested by collecting startle data from a group of four adult male Sprague-Dawley rats. The subjects were about 100 days old at the time of the testing and weighed 


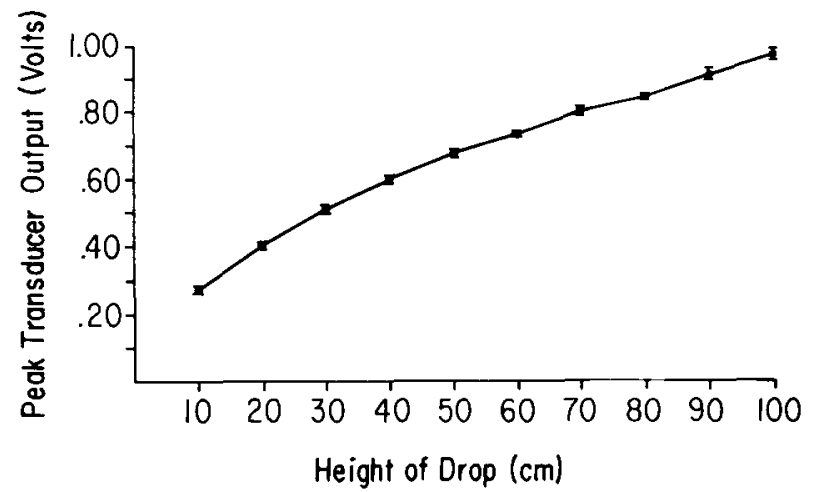

Figure 1. Mean output $( \pm$ SEM) of piezoelectric film startle transducer when a $1.2-\mathrm{g}$ weight was dropped upon it repetitively from varying heights.

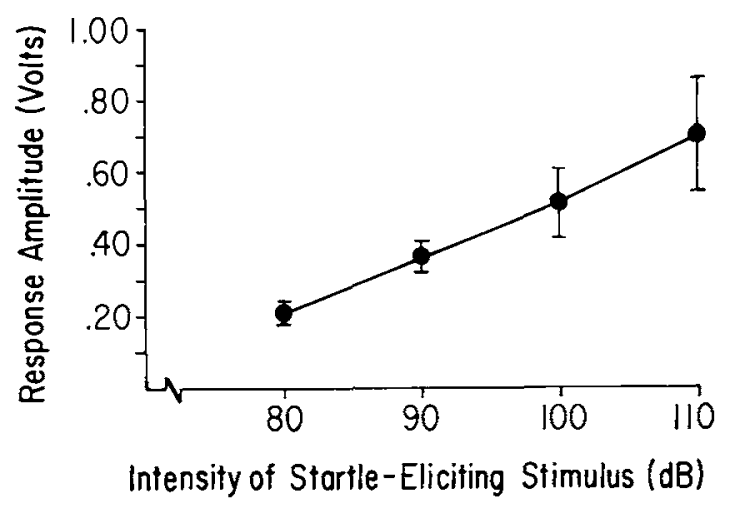

Figure 2. Mean startle amplitudes $( \pm$ SEM) obtained from a group of four rats exposed to white-noise bursts of several different intensities, as measured with the piezoelectric film transducer.

between 300 and $400 \mathrm{~g}$. The rats were tested twice, on days interspersed among the calibration test sessions described above. One startle test session was conducted 1 week after the first calibration session, and the second startle test session was conducted 1 week after the second calibration session.

In each test session, the subjects were individually exposed to a series of white-noise bursts (rise-fall time: $0.1 \mathrm{msec}$; duration: $25 \mathrm{msec}$ ) at each of four intensities $\left(80,90,100\right.$, and $110 \mathrm{~dB}$ re: $\left.0.0002 \mathrm{dyne} / \mathrm{cm}^{2}\right)$. The order of presentation of the four stimulus intensities was randomized within a block of trials, and 10 trial blocks were presented, resulting in 40 trials in all. The intertrial interval was $20 \mathrm{sec}$. Rise-fall time was controlled by a Grason-Stadler Company electronic switch (Model 829D), and stimulus intensity was varied manually with a General Radio decade attenuator (Type 1450TB) and measured with a General Radio precision soundlevel meter (Type 1561). The startle-eliciting stimulus was generated by a General Radio random-noise generator (Type 1390-B), was amplified by a David Hafler Company power amplifier (Model DH-500), and was presented to the subjects through four piezoelectric tweeters wired in series. The tweeters were mounted on a wall of the test chamber at a height that placed them level with the cage and $15 \mathrm{~cm}$ from one of its sides.
Stimulus duration was controlled by a Grass stimulator (Model S88). Response amplitude was measured with the Coldspring A/D converter, as described above, except that the response-window duration was reduced to $100 \mathrm{msec}$, which began with the presentation of the startle-eliciting stimulus.

For each session, mean startle amplitude was computed across trials for each intensity for each subject. Grand means were then computed across subjects and test sessions for each intensity. These data are depicted in Figure 2. It can be seen that the mean startle amplitude for each of the four intensities of startle-eliciting stimuli used was within the linear range of the transducer. Furthermore, a linear relationship between startle amplitude and stimulus intensity is apparent. A Pearson product-moment correlation coefficient computed from these data demonstrated this to be reliable $[\mathrm{r}(2)=.99$, $p<.01]$. These data are similar to functions relating startle amplitude to startle-eliciting stimulus intensity in the rat obtained by other researchers (e.g., Stitt, Hoffman, \& Marsh, 1976).

\section{CONCLUSIONS}

The piezo film material has shown itself to be a simple, linear transducer for startle. Its high output allows it to be used directly with a variety of measuring devices, and its low cost increases its utility. Although the focus of the present discussion has been on its application as a startle transducer, there is no reason why it cannot be adapted for use in a variety of other situations in which it is necessary to record small-animal movement. For example, if several pieces of the piezo film were attached to different parts of a large, thin sheet of Plexiglas, it could potentially be utilized as a smallanimal activity monitor in an open field situation.

\section{REFERENCE NOTE}

1. Foss, J. A., \& Ison, J. R. Personal communication, 1982.

\section{REFERENCES}

Fleshler, M. Adequate acoustic stimulus for startle reaction in the rat. Journal of Comparative and Physiological Psychology, 1965, 60, 200-207.

Hoffman, H. S., \& Flebhler, M. An apparatus for the measurement of the startle response in the rat. American Journal of Psychology, 1964, 77, 307-308.

Stitr, C. L., Hofrman, H. S., \& Marsh, R. R. Interaction versus independence of startle-modification processes in the rat. Journal of Experimental Psychology: Animal Behavior Processes, 1976, 2, 260-265.

White, E. H., \& Horlington, M. An apparatus for measuring startle response and motor activity in rats. Medical and Biological Engineering, 1969, 7, 325-327.

\section{NOTE}

1. Circuit diagrams for the sensor can be obtained by writing to John A. Foss, Department of Psychology, University of Rochester, River Campus Station, Rochester, New York 14627.

(Manuscript received May 6, 1983;

revision accepted for publication September $23,1983$. 